Article

\title{
A Surfactant Directed Microcrystalline Cellulose/Polyaniline Composite with Enhanced Electrochemical Properties
}

\author{
Mahnaz M. Abdi ${ }^{1, *(\mathbb{C})}$, Paridah Md Tahir ${ }^{2}$, Rawaida Liyana ${ }^{2}$ and Ramin Javahershenas ${ }^{3}$ ([) \\ 1 Young Researchers and Elite Club, Islamshahr Branch, Islamic Azad University, Islamshahr 3314767653, Iran \\ 2 Institute of Tropical Forestry and Forest Products, Universiti Putra Malaysia, Serdang 43400, Malaysia; \\ parida@upm.edu.my (P.M.T.); rayda_beep91@yahoo.com (R.L.) \\ 3 Department of Organic Chemistry, Faculty of Chemistry, Urmia University, Urmia 5756151818, Iran; \\ jshbco@yahoo.com \\ * Correspondence: mahnaz@upm.edu.my or mahnaz131@gmail.com; Tel.: +98-9228496079 \\ Academic Editor: Janet L. Scott \\ Received: 4 July 2018; Accepted: 24 August 2018; Published: 26 September 2018

\begin{abstract}
In this study a cationic surfactant, cetyltrimethylammonium bromide (CTAB), was used as a soft template for in situ chemical polymerization of aniline on the surface of microcrystalline cellulose (MCC). The morphology of the wire-like and porous nanostructure of the resulting composite was highly dependent on the MCC and CTAB concentrations. The effect of the MCC and $\mathrm{CTAB}$ concentrations on the electrochemical and morphological properties of the polyaniline (PAni) nanocomposite was studied. Cyclic voltammograms of modified PAni/MCC/CTAB electrode displayed a high current response and the effect of scan rate on the current response confirmed a diffusion controlled process on the surface of the electrode that makes it suitable for sensor applications. The overlapping characteristic peaks of pure PAni and MCC caused peak broadening at $3263 \mathrm{~cm}^{-1}$ in the IR spectra of PAni/MCC/CTAB nanocomposite that revealed the interaction between NH of PAni and $\mathrm{OH}$ group of MCC via electrostatic interactions. The addition of MCC to PAni through chemical polymerization decreased the thermal stability of composite compared to pure PAni. Lower crystallinity was observed in the XRD diffractogram, with 2 theta values of 22.8, 16.5, and 34.6 for PAni/MCC, confirming the formation of PAni on the MCC surface.
\end{abstract}

Keywords: conducting polymers; microcrystalline cellulose; chemical polymerization; modified electrode

\section{Introduction}

Natural cellulose fibers contain crystalline and amorphous domains. Microcrystalline ellulose (MCC) can be synthesized by different processes such as reactive extrusion, enzyme mediated, and acid hydrolysis in that way the amorphous regions are removed and the crystalline domains remain. The hydroxyl groups covering the cellulose surface and orderly arrangement of molecules in MCC allows the cellulose to react well with a variety of materials, including conducting polymers [1]. There are numerous composites of cellulose and its derivatives with synthetic polymers and biopolymers that have been used frequently in different applications such as biomedical applications [2], sensors and actuators, [3] and nanocomposites with good tensile strength [4].

Conducting polymers are some of the most studied materials that have been used to modify the crystals and fibers of cellulose. The poor solubility and dispersibility of conducting polymers in common organic solvents are limiting factors for them to be used in different applications $[5,6]$. On the other hand the electron transfer in a bulky polymer is relatively slow which limits its application in 
sensors. A composite of conducting polymers and hydrophilic cellulosic materials with high surface area, tensile strength and good water-dispersibility can overcome these problems [7].

The nanocomposites of cellulose and conducting polymers synergistically combine the electronic characteristics of the conducting polymers with the structural advantages of biobased cellulose, making them useful for different applications [8]. The good interaction between binding sites on the cellulose and cationic species make cellulose a good candidate for sensor devices. Permselective membranes for different species can be modified by a treatment process through changing the surface functionality of cellulose and its permselective properties [9]. A composite of microfibrillated cellulose (MFC) and PPy was prepared by chemical polymerization of pyrrole on the MFC in a hydrogel form [10]. The MFC/PPy nanocomposite with an open, porous structure of intertwined fibers exhibited an acceptable ion-exchange capacity for chloride ions. In recent year, the combinations of PAni and cellulose-based materials have been prepared by various methods to enhance phe hysical and structural properties of the resulting composites. Intrinsic conducting polymers and conductive nanocrystalline cellulose composite have been used as mediators to facilitate electron transfer between the electrode and analyte in biosensors $[4,11]$. In a study by Lee [4], crystalline nanocellulose-polpyrrole (CNC-PPy) was cast on a microfabricated interdigitated electrode and used as a glucose micro-biosensor. They showed that the compatibility of cellulose with the enzyme combined with the change in the resistance of the doped-PPy could enhance the sensitivity factor by as much as 20 .

In many studies composites of PAni/cellulose with enhanced physical and electrochemical properties were used in high capacity biodegradable batteries [1,12,13], chemical sensors [14], and electroactive paper [15]. In biosensor applications, crystalline nano-cellulose (CNC) was introduced into the polymer structure to provide a larger active surface area and higher specific strength [16].

A cholesterol biosensor based on polyaniline and gold nanocomposite was successfully fabricated using a seed-mediated method [12]. Liu et al. [17] engineered a flexible and electrically conductive nanocellulose-based polyaniline composite film. They reported that the composite film with a thickness of $50 \mu \mathrm{m}$ could be bent up to 180 degrees without breaking.

In the current research we used the cationic surfactant cetyltrimethylammonium bromide $(\mathrm{CTAB})$ as a soft template for aniline/MCC polymerization. There are some reports on the synthesis of conducting polymer nanostructures using surfactants as hard or soft templates to produce a porous material for sensing applications [18]. Using surfactants as template for making nano- and mesostructures can increase the penetration rate of the target molecules into the sensing area and decrease the response time in biosensors [19]. The effect of the CTAB and MCC concentration on the physical and electerochemical properties of the polymer was investigated to develop a nanocomposite with enhanced electrochemical properties for biosensor applications.

\section{Results and Discussion}

\subsection{Electrochemical Properties}

Cyclic voltammetry was done to optimize conditions for nanocomposite preparation and find a composition with the highest anodic current. The effect of MCC and CTAB concentration on the electrochemical properties of nanocomposite was determined using $\mathrm{CV}$ performed from -1.5 to $+1.5 \mathrm{~V}$ at a scan rate of $100 \mathrm{mV} \mathrm{s}^{-1}$. Figure $1 \mathrm{~A}$ depicts the cyclic voltammograms for pure polyaniline and the PAni-MCC composite prepared from solutions containing different Ani/MCC mass ratios. The cyclic voltammogram of the PAni-modied electrode showed anodic peaks at the potentials of 0.13 and $0.81 \mathrm{~V}$. A small oxidation peak observed at $0.13 \mathrm{~V}$ is associated with the conversion of the fully reduced leucoemeraldine base to the partially-oxidized emeraldine. As the potential increased the conversion of the emeraldine form to the fully oxidized pernigraniline form occurred at $0.81 \mathrm{~V}$, which is in agreement with the results reported by others [20]. The reduction of the electrically conductive emeraldine (EM) to the non-conducting leucoemeraldine (LEM) was observed at $-1.01 \mathrm{~V}$. 
The PAni-MCC nanocomposite-modified electrodes presented voltammograms with higher cathodic and anodic peaks (Figure 1A).
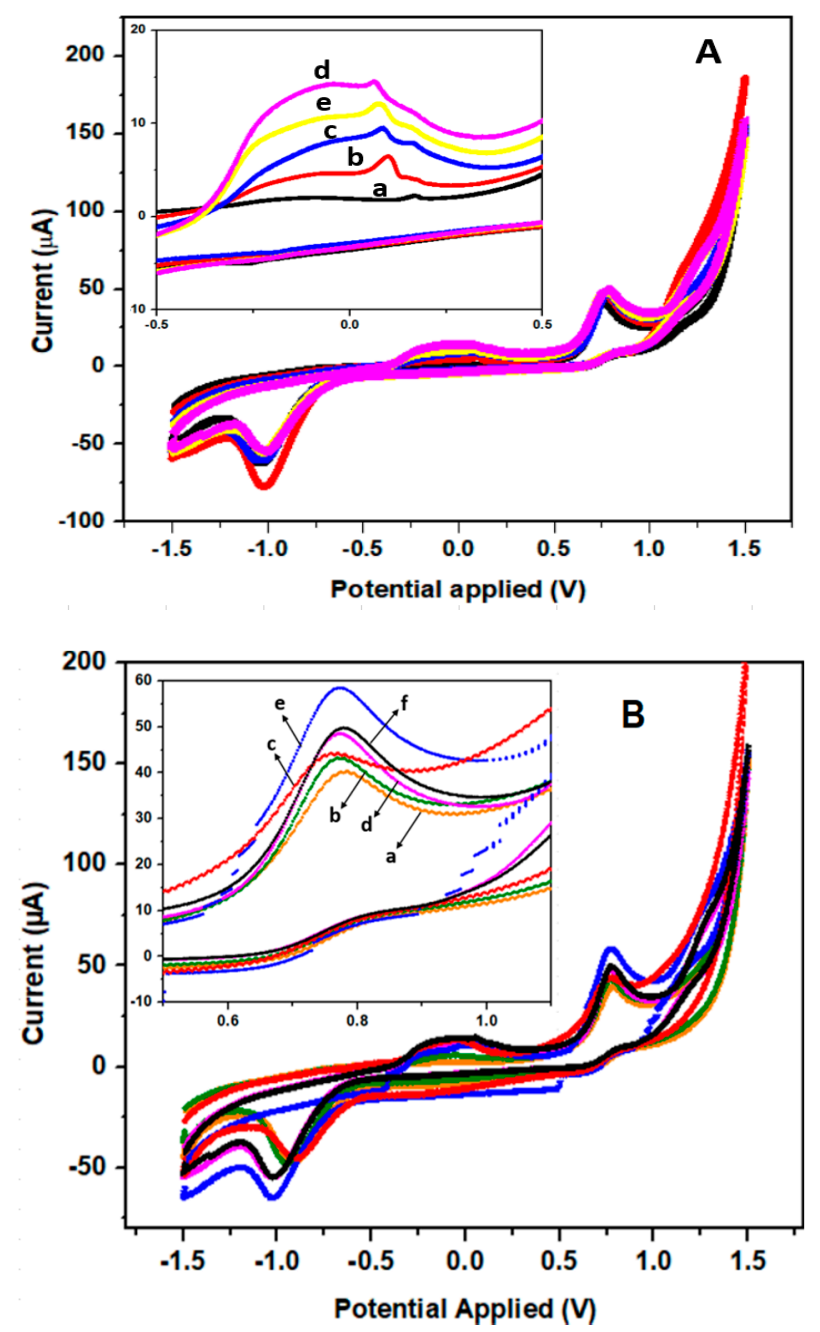

Figure 1. (A) Cyclic voltammograms of (a) pure PAni, PAni/MCC nanocomposite with mass ratio of Ani/MCC (b) 0.37/0.63, (c) 0.56/0.44, (d) 0.74/0.26, (e) 0.93/0.07, (B) Cyclic voltammograms of PAni/MCC/CTAB nanocomposite prepared from solutions containing different concentrations of CTAB ranging from: (a) $0 \mathrm{cmc}$ (PAni/MCC), (b) $4 \mathrm{cmc}$, (c) $6 \mathrm{cmc}$, (d) $8 \mathrm{cmc}$, (e) $10 \mathrm{cmc}$ (f) $12 \mathrm{cmc}$. Inset: enlarged anodic peak.

It is known that PAni is conductive only in acidic media; however, PAni composites must be able to be used in neutral media as biosensors. On the other hand it has been shown that the ionization of the carboxyl groups of cellulose in a neutral medium will produce an excess net negative charge on the PAni/cellulose composite. In order to compensate the extra negative charges the composite undergoes protonation which increases the proton concentration inside the composite compared to the external solution; consequently PAni/cellulose composite shows conductivity even under neutral conditions [21]. As it can be seen from Figure 1A, by increasing the MCC content (mass ratio of Ani/MCC from $0.93 / 0.07$ to $0.74 / 0.26$ ) in the nanocomposite, the oxidation peaks at the potentials of 0.13 and $0.81 \mathrm{~V}$ increased and the composition of $0.74 / 0.26$ mass ratio $(0.16 \mathrm{M}$ aniline) exhibited the highest current response of 14.4 and $49.7 \mu \mathrm{A}$, respectively. This was possibly due to the protonation of PAni in the presence of a higher amount of MCC. Another possible reason is due to the capability of MCC to produce nanostructures with a higher surface area and porosity that facilitate the electron transfer. The fibrous and porous structure of PAni/MCC (compared with bulky PAni) which was 
observed in FESEM images justified a higher electron transfer of the composite. After the addition of MCC content accounting for more than $26 \%$ of the composite, the cyclic voltammogram of PAni/MCC presented a lower anodic peak and the nanocomposite with a mass ratio of $0.37 / 0.63(0.08 \mathrm{M}$ aniline $)$ exhibited the lowest redox current. This was probably due to the insulating nature of cellulose that restricted the electron transfer in the polymer chain. All voltammograms were taken in $0.1 \mathrm{M}$ PBS of pH 7.0 with a potential scan rate of $100 \mathrm{mV} \mathrm{s}^{-1}$ at room temperature.

$\mathrm{CTAB}$ content optimization was further conducted to study the effect of CTAB on the electrochemical properties of the nanocomposites. Figure $1 \mathrm{~B}$ shows the voltammograms of PAni/MCC/CTAB nanocomposite prepared from solutions containing different concentrations of CTAB ranging from $4-12 \mathrm{cmc}$. The concentration of Ani was $0.16 \mathrm{M}$ and the mass ratio of Ani/MCC was kept fixed at $0.74 / 0.26$.

The enlarged anodic peak is displayed in the inset graph for more clarification. The nanocomposites presented an increment in the anodic peaks after addition of CTAB and the nanocomposite prepared from $10 \mathrm{cmc}$ of $\mathrm{CTAB}$ showed the highest redox current of $58.7 \mu \mathrm{A}$. It was found that CTAB has a significant effect on the formation of the nanostructure of the PAni composite which is related to its function as a soft template for the polymerization of aniline by the self-assembly of the cationic surfactant and monomer. It has been shown that the type of surfactant and its concentration play important role in controlling the diameter of nanoparticles by partially solubilizing the template [22]. Figure 2 shows CVs of PAni/MCC/CTAB-modified electrode with different scan rate. The nanocomposite was prepared from a solution containing Ani/MCC with mass ratio of $0.74 / 0.26$ and $10 \mathrm{cmc}$ CTAB. As it can be seen that both the oxidation and reduction peaks increased with the increasing scan rate. Most cycles showed both the oxidation and reduction peaks, in which the anodic potential shifted towards the lower potential and the cathodic potential slightly shifted to the more negative value as the scan rate increased. The concentration profile around the electrode is influenced by the rate of potential scanning. This phenomenon can change the rate of charge transport indicating the diffusion controlled nature of a system. The anodic and cathodic peaks were found to be proportional to the square root of the scan rate, which clearly indicated the occurrence of a diffusion-controlled process (inset plot) [23].

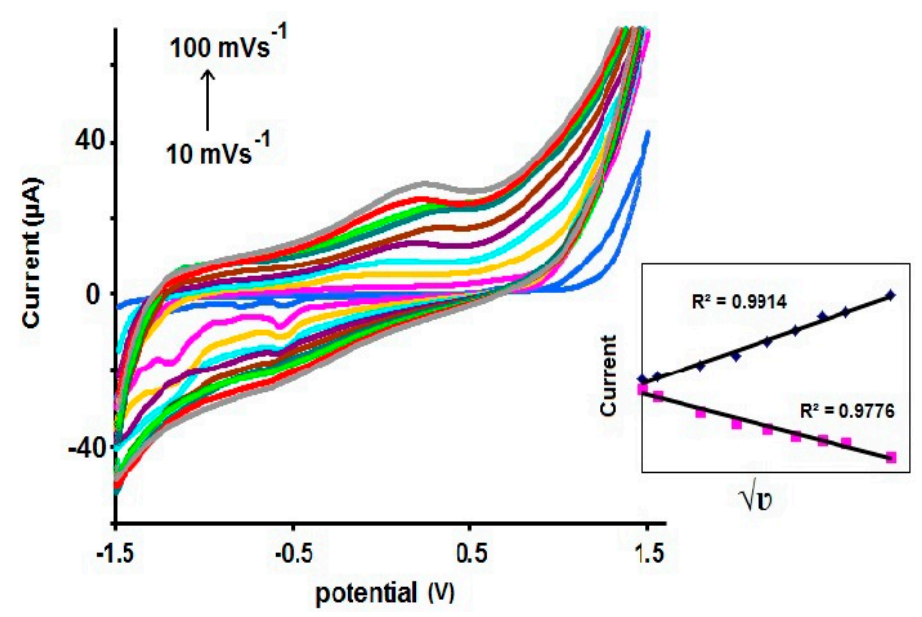

Figure 2. Cyclic voltammograms of PAni/MCC/CTAB-modified electrode with different scan rate. Inset: plot of oxidation and reduction peak current against square root of scan rate. All voltammograms were taken in $0.1 \mathrm{M}$ PBS of $\mathrm{pH} 7.0$ with a potential scan rate of $100 \mathrm{mV} \mathrm{s}^{-1}$ at room temperature. 


\subsection{Chemical, Morphological and Structural Characterizations}

\subsubsection{Fourier Transform Infrared (FTIR) Spectroscopy}

Figure 3 compares the FT-IR spectra of pure PAni, MCC, and PAni/MCC/CTAB nanocomposite. Pure doped-PAni showed characteristic peaks at $3209 \mathrm{~cm}^{-1}, 2938 \mathrm{~cm}^{-1}$ associated with the N-H stretches and $\mathrm{C}-\mathrm{H}$ stretch, respectively. The characteristic peaks assigned to the $\mathrm{C}=\mathrm{C}$ stretches and $\mathrm{C}=\mathrm{N}$ bond in the polyaniline units appeared at $1660 \mathrm{~cm}^{-1}$ and $1542 \mathrm{~cm}^{-1}$, respectively. The aromatic stretching and C-C bending vibrations appeared at $1408 \mathrm{~cm}^{-1}$ and $1258 \mathrm{~cm}^{-1}$ [24,25]. In the MCC structure, a strong $\mathrm{O}-\mathrm{H}$ stretching absorption band occurred at $3343 \mathrm{~cm}^{-1}$ and the peak appearing at $2906 \mathrm{~cm}^{-1}$ represented the aliphatic $\mathrm{C}-\mathrm{H}$ stretching. The sharp peak of $\mathrm{C}-\mathrm{O}$ stretching of the primary alcohol group $\left(-\mathrm{CH}_{2} \mathrm{OH}\right)$ appeared at $1040 \mathrm{~cm}^{-1}$, whereas the peak at $1331 \mathrm{~cm}^{-1}$ showed the $\mathrm{C}-\mathrm{O}$ bending. The spectra of PAni/MCC/CTAB nanocomposite (prepared from Ani/MCC mass ratio $=0.74 / 0.26$ and $10 \mathrm{cmc}$ surfactant) is also presented in Figure 3. The spectrum revealed both characteristic peaks of pure PAni and MCC, thus demonstrating the polymerization of aniline on the surface of MCC. The overlapping characteristic peaks of pure PAni at $3200 \mathrm{~cm}^{-1}$ and MCC at $3331 \mathrm{~cm}^{-1}$ caused peak broadening at $3288 \mathrm{~cm}^{-1}$ in the IR spectra of PAni/MCC/CTAB nanocomposite revealing the interaction between $\mathrm{NH}$ of PAni and $\mathrm{OH}$ group of MCC via electrostatic interactions [26,27]. The nanocomposite of PAni/MCC/CTAB showed lower O-H band intensity compared to the pure MCC, which might due to the crystalline cellulose being covered by PAni, which is in agreement with other studies [28,29]. In the $\mathrm{CTAB}$ molecule the $\mathrm{CH}_{3}$ asymmetric stretching $\left(v_{\mathrm{as}}\right)$ and $\mathrm{CH}_{3}$ symmetric stretching $\left(v_{\text {sym }}\right)$ frequencies were observed at 2915 and $2849 \mathrm{~cm}^{-1}$, respectively. The $\mathrm{N}-\mathrm{CH}_{3}$ stretching appeared as a weak shoulder at $2949 \mathrm{~cm}^{-1}$. The presence of a peak at $2936 \mathrm{~cm}^{-1}$ in the nanocomposite confirmed the existence of $\mathrm{CTAB}$ in the composite structure. The $\mathrm{C}-\mathrm{N}$ stretching vibration observed at $909 \mathrm{~cm}^{-1}$ in CTAB shifted to $1010 \mathrm{~cm}^{-1}$ in PAni/TA/CTAB nanocomposite $[19,30]$.

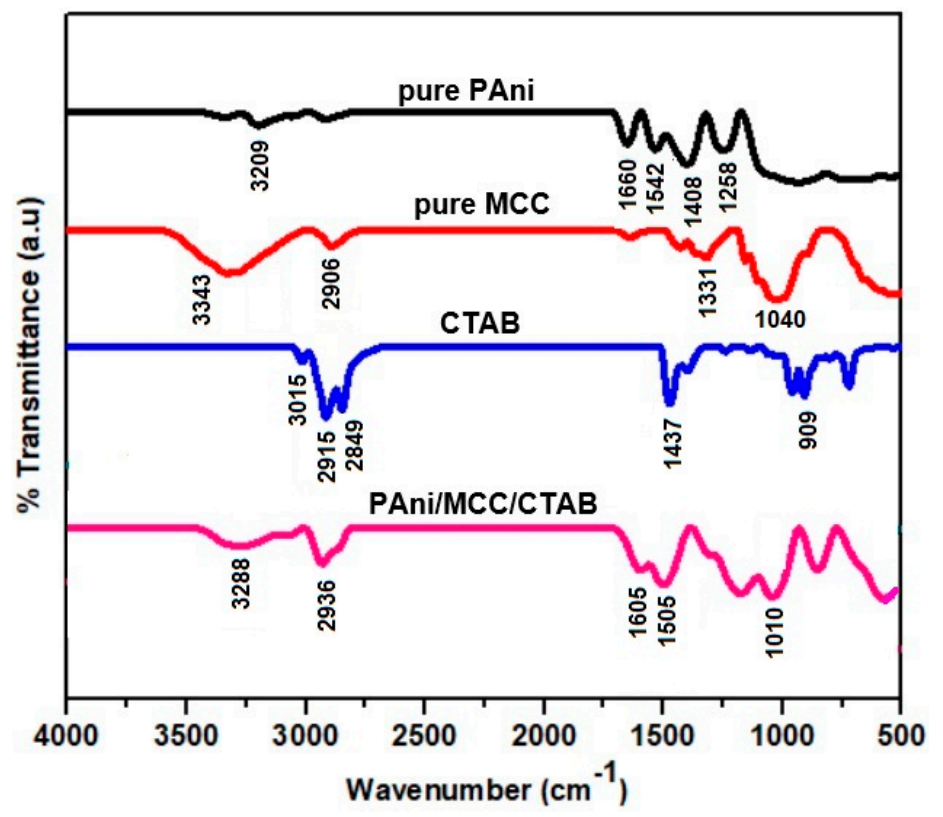

Figure 3. FT-IR spectra of pure PAni, pure MCC, CTAB, and PAni/MCC/CTAB nanocomposite.

The stretching vibration of the benzoid form of PAni appearing at $1505 \mathrm{~cm}^{-1}$ in the PAni/MCC/CTAB nanocomposite showed a uniform formation of PAni on the nanofibrous structure. Based on the combined FTIR results, the following structure (Scheme 1) is proposed for PAni/MCC/CTAB nanocomposite. 

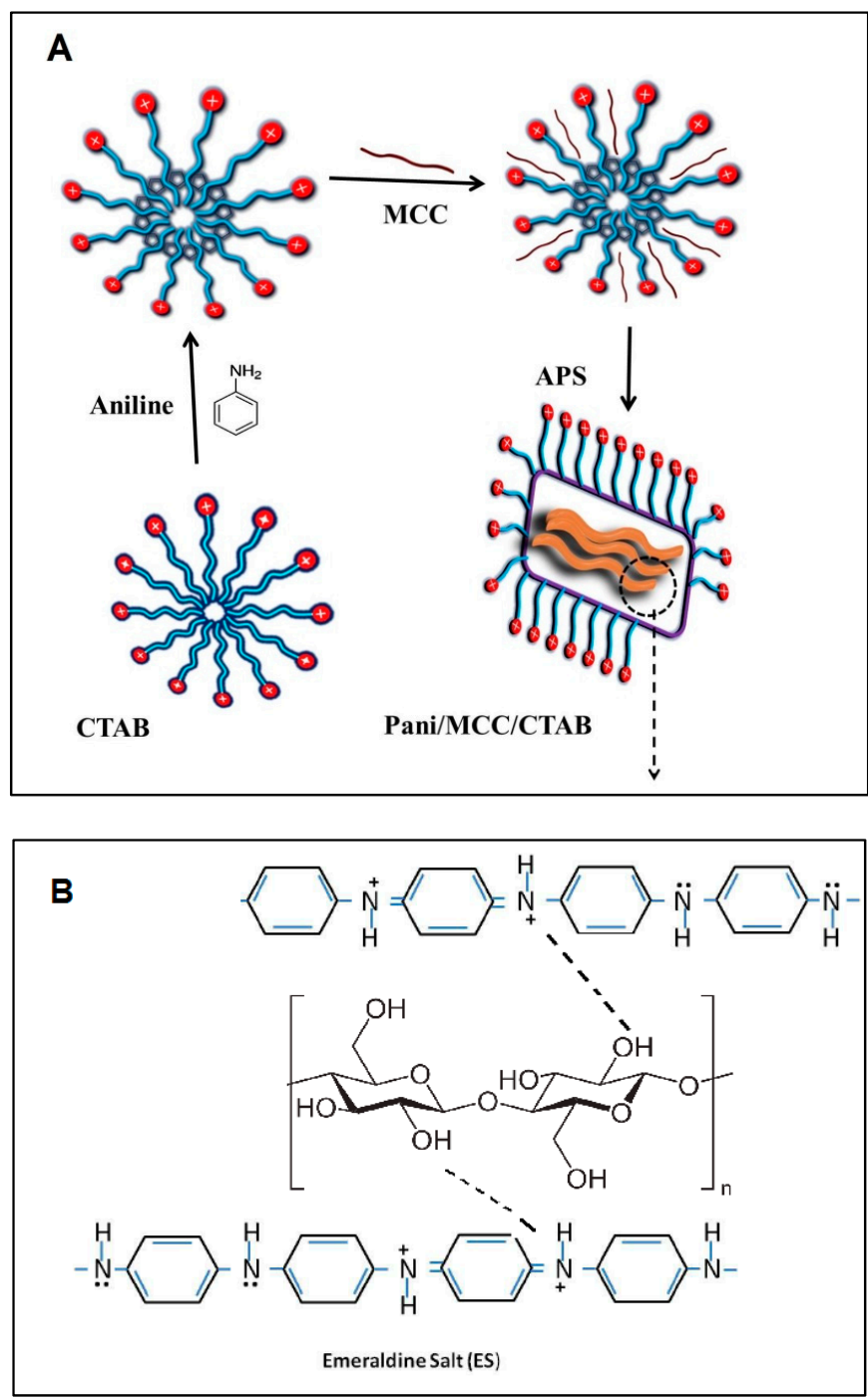

Scheme 1. (A) View of chemical polymerization of aniline in the presence of MCC and CTAB and (B) possible intraction between cellulose and PAni.

\subsubsection{Morphology}

FESEM micrographs of pure PAni and PAni/MCC with different MCC content are presented in Figure 4. Pure PAni showed a bulky, granular, and dense structure (Figure 4a) but a remarkable changes from bulky and shapeless structure to spherical particles was observed upon addition of MCC to the polymer (Figure $4 \mathrm{~b}$ ), proving the polymerization of aniline on the cellulose surface [31]. The features of the PAni/MCC composite changed slightly to a rod-like structure on further addition of MCC whereupon the fibers become longer with less agglomeration of particles (Figure 4c). The composite prepared from $0.16 \mathrm{M}$ aniline (Figure $4 \mathrm{~d}$ ) showed a fibrous feature with a high porosity nanostructure, which could be beneficial for biomolecule immobilization. The proper mixing of MCC and PAni combines the nanostructure advantage of biopolymer and the electrical properties of PAni explaining the higher anodic current obtained for the composite. The addition of extra amounts of cellulose (Figure 4e) formed intertwined fibers; with nodular agglomerates of particles in some parts of the nanocomposite. It seems that the composite with higher amount of MCC presented a smooth and uniform surface fiber displaying crystalline and insulating cellulose with lower electroactive property that justifies the lower redox current in the CV graph. 


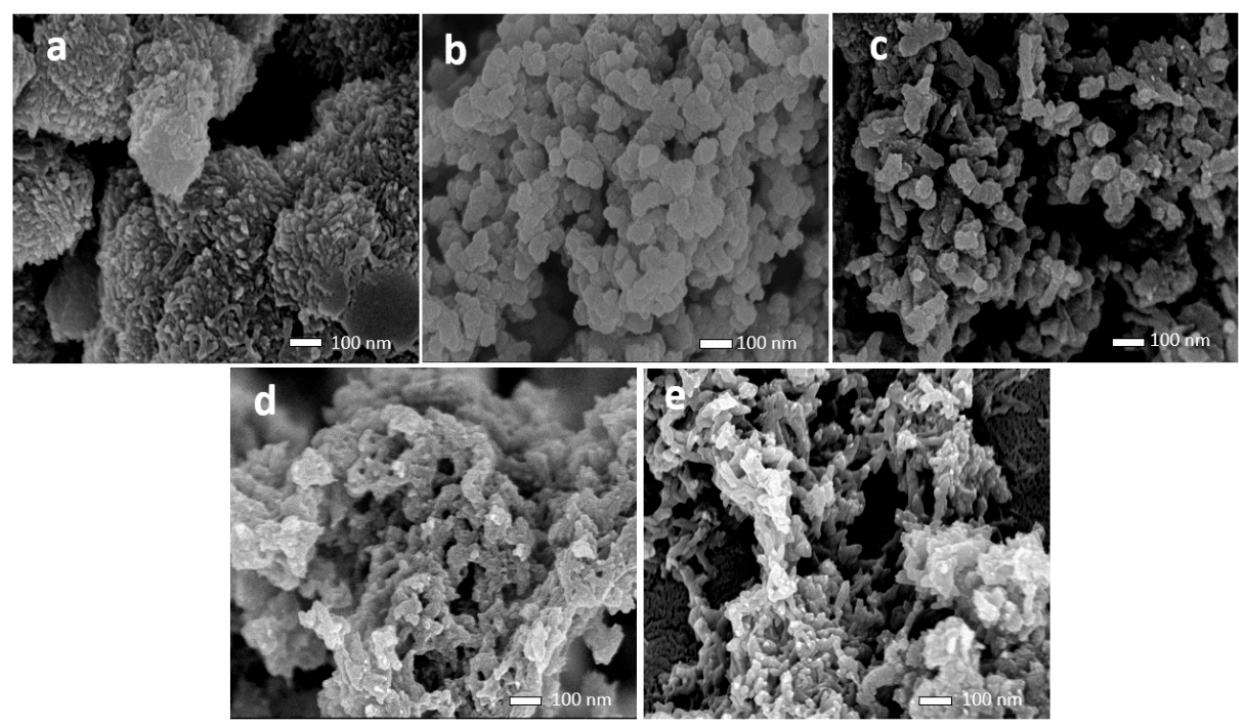

Figure 4. FESEM micrographs of (a) pure PAni, and PAni/MCC nanocomposite prepared from solutions containing different mass ratio of Ani/MCC of (b) $0.37 / 0.63$; (c) $0.56 / 0.44$; (d) 0.74/0.26; (e) $0.93 / 0.07$.

Figure 5 presents the morphology of PAni/MCC/CTAB prepared from solutions containing $0.16 \mathrm{M}$ aniline (Ani/MCC mass ratio of 0.74/0.26) and different concentrations of CTAB. After adding 4 and $6 \mathrm{cmc}$ surfactant, a few particles with wire-like structure were observed (Figure 5a,b). It has been proved the cationic surfactant of CTAB works as a soft template to tailor the shape of the polymer into desired nanostructures in such a way that above a critical concentration of surfactants, they assemble into micelles, spherical or cylindrical structures [32]. In our study, by increasing CTAB to t8 and $10 \mathrm{cmc}$, intertwined wire and ribbon-like structures were observed (Figure 5c,d) providing more porous structures with less agglomerated particles which could facilitate the electron transfer between electrode and mediator.
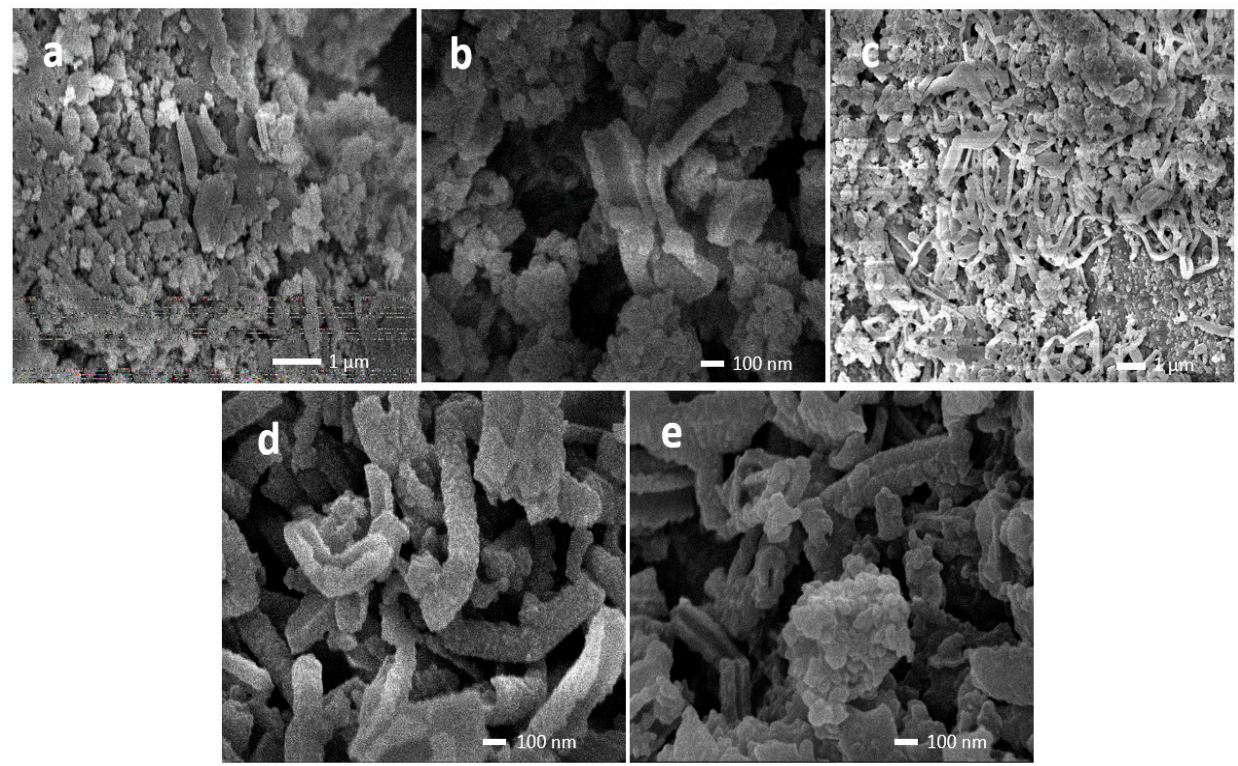

Figure 5. FESEM micrographs of PAni/MCC/CTAB nanocomposite prepared from different concentration of CTAB (a) $4 \mathrm{cmc}$; (b) $6 \mathrm{cmc}$; (c) $8 \mathrm{cmc}$; (d) $10 \mathrm{cmc}$; (e) $12 \mathrm{cmc}$. 
At high concentrations of CTAB the ribbon-like particles formed were significantly shorter in length and twisted to produce half tube structures (Figure 5e,f).

\subsubsection{X-ray Diffraction Analysis}

Figure 6A shows the XRD pattern of PAni, and MCC. Pure PAni presented peaks at $2 \theta=20.5^{\circ}$ and $25.4^{\circ}$, ascribed to the repetition of the benzoid and quinoid rings in the PAni chains [33] confirming polyaniline was in the form of emeraldine salt [34].
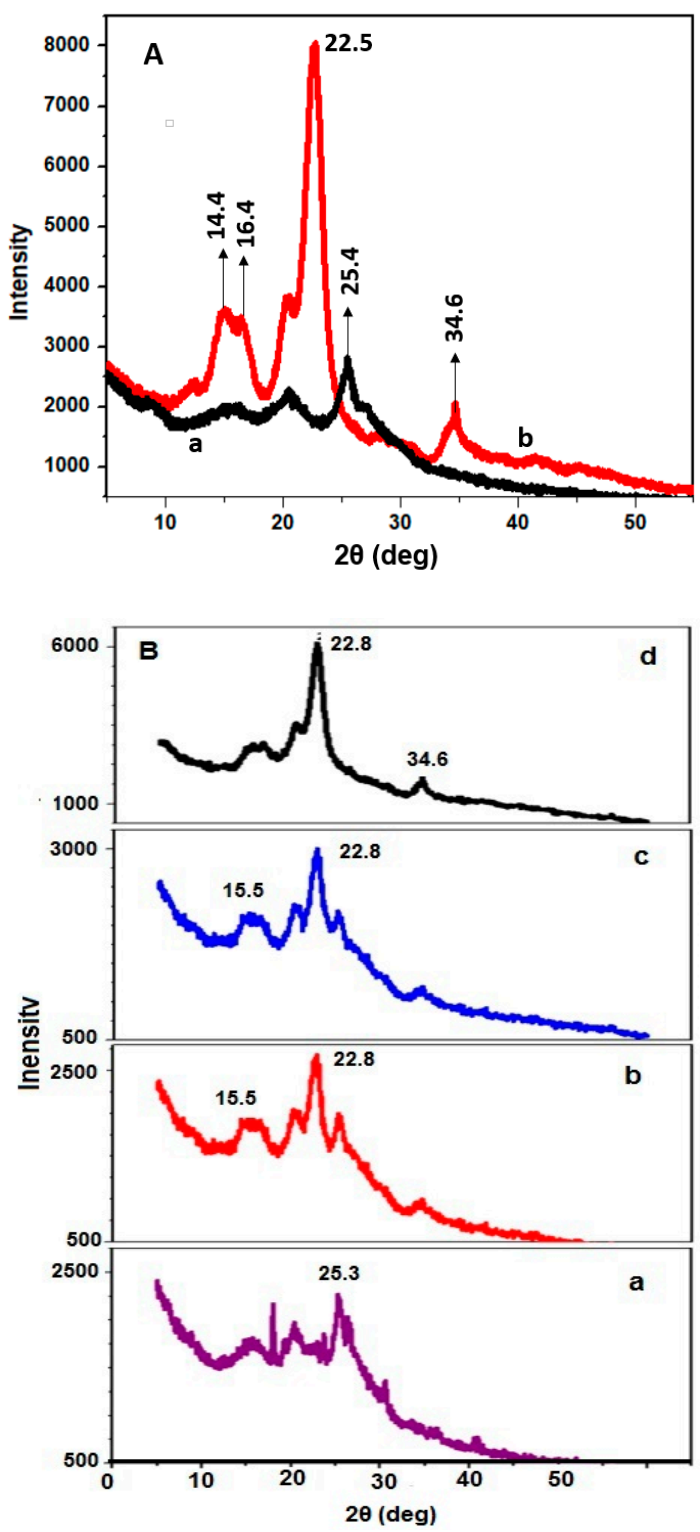

Figure 6. (A) X-ray diffractrograms of (a) pure PAni, and (b) MCC; (B) PAni/MCC prepared from different mass ratio of Ani/MCC (a) 0.93/0.07; (b) 0.74/0.26; (c) 0.56/0.44; (d) 0.37/0.63.

The XRD diffractogram of MCC (Figure $6 \mathrm{~A}(\mathrm{~b})$ ) showed the highest intensity at $2 \theta=22.5^{\circ}$, and two small peaks at $2 \theta=16.4^{\circ}$ and $14.6^{\circ}$ correspond to the Miller indices of (200), (110) and (1-10), respectively [35]. Pure MCC also displayed a small peak at $2 \theta=34.6^{\circ}$, all confirming the typical pattern for cellulose I.

The effect of MCC content on the crystallinity aspects was studied and Figure 4B compares the XRD diffractograms of PAni/MCC nanocomposites with different mass ratios of Ani/MCC. 
The nanocomposite with the highest amount of MCC (Figure 6B(d)) exhibited the highest intensity peak at $22.8^{\circ}$ and a small peak at $2 \theta=34.6^{\circ}$. The nanocomposite with high PAni content (Figure $6 \mathrm{~B}(\mathrm{a})$ ) exhibited the highest peak at 2 theta of $25.3^{\circ}$ indicating the superior effect of PAni on the crystallinity of the nanocomposite. The results are in agreement with those obtained by others [17].

\subsubsection{Thermogravimetry Analysis (TGA)}

The TGA plots of pure PAni, PAni/MCC (Ani/MCC mass ratio of 0.74/0.26), and PAni/MCC/CTAB (Ani/MCC mass ratio of 0.74/0.260 and $10 \mathrm{cmc}$ of CTAB) are presented in Figure 7. Doped polyaniline typically exhibits three major weight loss steps. The first weight loss attributed to the removal of moisture starts at $56{ }^{\circ} \mathrm{C}$ and continues rapidly up to $150{ }^{\circ} \mathrm{C}$. The second step, due to the dopant decomposition, gradually occurred from $158^{\circ} \mathrm{C}$ to $353{ }^{\circ} \mathrm{C}$. The last weight loss happened around $457^{\circ} \mathrm{C}$ to $600{ }^{\circ} \mathrm{C}$ and is attributed to decomposition of the polymer backbone in agreement with the results of others [36]. The incorporation of crystalline cellulose into the PAni increased the crystallinity of the composite, where more energy was required to evaporate water/acid from the polymer chain [37] resulting a higher degradation temperature for PAni/MCC in the first step. In other words, PAni chains doped with acid in a more crystalline structure would need more energy for removing acid from well arranged polymer chains. Therefore, the second weight loss of PAni/MCC with higher crystallinity shifted to a higher temperature. The pyrolysis of cellulose content in PAni/MCC composite was observed over the range from $230{ }^{\circ} \mathrm{C}$ to $350{ }^{\circ} \mathrm{C}$ [38] that is higher compared to that of pure cellulose, suggesting the protection of PAni over the surface of cellulose [39].

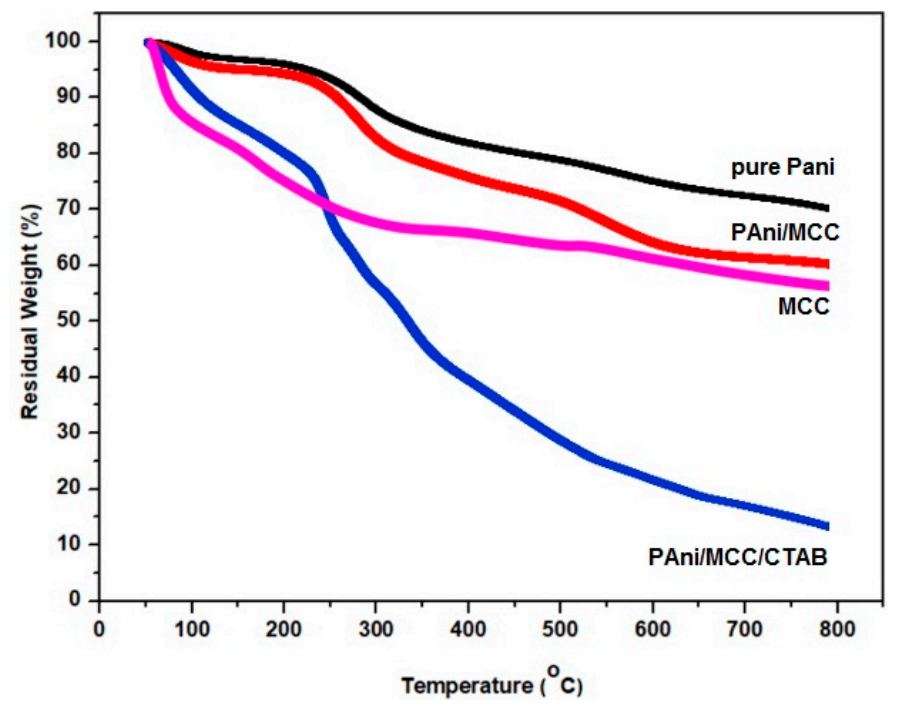

Figure 7. TGA curves of Pure PAni, PAni/MCC, MCC, and PAni/MCC/CTAB nanocomposite.

Pyrolysis of cellulose in microcrystalline cellulose occurred at a higher temperature compared to that of nanocrystalline cellulose [17]. This could be due to the bigger size, higher crystallinity, and higher polymerization degree of MCC.

A lower maximum decomposition temperature was observed for the PAni/MCC nanocomposite compared to that for pure PAni, confirming the interactions between the two constituents of the composite [38]. The thermal stability of PAni was noticeably decreased by incorporating MCC. However, adding CTAB to the composite resulted in lower thermal stability of the nancomposite and PAni/MCC/CTAB nanocomposite decomposed at a lower temperature due to the calcination of CTAB up to $250{ }^{\circ} \mathrm{C}$ [40]. CTAB transformations during calcination of CTAB up to $\sim 250{ }^{\circ} \mathrm{C}$ is well known and it completely decomposed where there is no trace of CTAB at temperatures from $200{ }^{\circ} \mathrm{C}-500{ }^{\circ} \mathrm{C}[40]$. 


\section{Materials and Methods}

\subsection{Materials}

Microcrystalline cellulose (MCC) was provided by R\&M Chemicals (Essex, United Kingdom) and was partially soluble in dilute mineral acids. Aniline used as monomer, purchased from Sigma Aldrich Chemie $\mathrm{GmbH}$ (Dramstadt, Germany), was distilled under reduced pressure at $131{ }^{\circ} \mathrm{C}$ and stored at $3{ }^{\circ} \mathrm{C}$ in the refrigerator until used. Ammonium persulphate (APS) as oxidant, hydrochloric acid ( $\left.\mathrm{HCl}\right)$ and phosphate buffer solution (PBS) were provided by R\&M Chemicals. Cetyltrimethylammonium bromide (CTAB) was purchased from Fluka BioChemika (Munich, Germany) and a screen printed carbon electrode (SPCE) was obtained from DropSens (Asturias, Spain). All chemicals were of analytical reagent grade and used without further purification.

\subsection{Preparation of the Nanocomposite and Modified Electrode}

Various amounts of aniline were dissolved in hydrochloric acid $(\mathrm{HCl}) 1 \mathrm{M}$ in an ice bath. In a separate conical flask MCC was dispersed in deionized water (DIW) and stirred moderately until the mixture reached homogeneity. Different concentration of CTAB ranging from $4-12 \mathrm{cmc}(1 \mathrm{cmc}$ of CTAB $=0.87 \mathrm{mM}$ ) was prepared in the DIW and then added into the Ani solution. Then, the Ani/CTAB solution was added into the MCC solution and the mixture was stirred at $0-5{ }^{\circ} \mathrm{C}$ for $5 \mathrm{~min}$ in the ice bath and left standing for $10 \mathrm{~min}$. The concentration of aniline was adjusted in the range of 0.08 to $0.2 \mathrm{M}$ and the mass ratio of Ani/MCC was selected as $0.37 / 0.63,0.56 / 0.44,0.74 / 0.26$, and $0.93 / 0.07$ in final mixture. The concentration of CTAB was in the range of $4-12 \mathrm{cmc}$. Critical micelle concentration, known as cmc, is the concentration of surfactants above which micelles form and all additional surfactants added to the system go to micelles. The solution of APS was added drop wise to the mixture in which the molar ratio of APS and aniline was always kept at 1:1. The mixture was kept stirring for $30 \mathrm{~min}$ and let it rest at room temperature for $18 \mathrm{~h}$. The green emeraldine polyaniline nanocomposite (PAni/MCC/CTAB) precipitate was filtered off and washed with distilled water at least 3 times through centrifugation till supernatant become clear. The precipitate was dried in the oven at $60{ }^{\circ} \mathrm{C}$ for $6 \mathrm{~h}$. Pure PAni was prepared at the same condition for comparison purpose. Composite of PAni/MCC was prepared in the same manner just without surfactant.

In order to prepare a modified electrode, $1 \mathrm{mg}$ of the dried sample was dispersed in $2 \mathrm{~mL}$ DIW and ultra-sonicated for $15 \mathrm{~min}$ to get a stable suspension. To obtain a homogeneous and uniform sample surface on the electrode, $10 \mu \mathrm{L}$ of suspension was dropped onto the screen-printed electrode (SPE), by drop casting followed by drying at room temperature.

\subsection{Methods}

The electrochemical measurements were conducted using an Autolab 204 potentiostat connected to a PC and controlled by Nova software version 2.11 (Metrohm, Utrecht, The Netherlands). Field Emission Scanning Electron Microscopy (FESEM) characterization was done by using JEOL JSM-7600F FESEM Microscope purchased from JEOL Ltd. (Tokyo, Japan) using the secondary detector, 7.0 probe size and $5.0 \mathrm{kv}$ acceleration voltages. Powder XRD data were carried out on a PANanalytical EMPYREAN system (Royston, UK) at $4.0 \mathrm{~kW}$ power supply, $100 \mathrm{~mA}$ current flow and $60 \mathrm{kV}$ operation voltage, and with $\mathrm{Cu} \mathrm{K} \alpha$ radiation $(\lambda=1.54 \AA$ ). The thermal gravimetric analysis of samples was done using a TGA/SDTA 851 system (Mettler Toledo, Ohio, USA) under constant heating rate $\left(10^{\circ} \mathrm{C} / \mathrm{min}\right)$ and $\mathrm{N}_{2}$ atmosphere with the temperature range of $50-800^{\circ} \mathrm{C}$. FTIR analysis was done on the samples by using a Spectrum 100FT-IR spectrometer (Perkin Elmer, Waltham, MA, USA) equipped with Universal Attenuated Total Reflectance (UATR). The nanocomposite $(100 \mathrm{mg}$ ) was mixed with potassium bromide $(\mathrm{KBr})$ and compressed to form a crystalline, clear pellet and examined in the transmittance mode within a $4000-400 \mathrm{~cm}^{-1}$ range. 


\section{Conclusions}

In this work it has been shown that the physical and electrochemical properties of polyaniline were enhanced in the presence of MCC and the cationic surfactant CTAB. The SPE electrodes modified by PAni/MCC/CTAB using certain amounts of MCC and CTAB presented the uppermost redox currents that could be appropriate for sensor applications. The PAni/MCC/CTAB nanocomposite prepared from $10 \mathrm{cmc}$ of CTAB showed the highest redox current of $58.7 \mu \mathrm{A}$, while PAni/MCC prepared from same composition exhibited an anodic current of $49.7 \mu \mathrm{A}$. The lamellar structure between the cationic surfactant and anion of the oxidizing agent, APS, serve them as a template to synthesize nanomaterials with high surface area and porosity. The electrochemical properties of doped PAni combined with the compatibility of cellulose make PAni/MCC composite a good candidate to be used in biodegradable batteries and biosensors. PAni/MCC/CTAB nanocomposite could represent a stable nanostructure platform for enzyme and biomolecule immobilization. The XRD and FESEM results confirmed the formation of s homogeneous PAni composite in the presence of CTAB and MCC

Author Contributions: Conceptualization, M.M.A.; Methodology, R.L., and R.J.; Formal Analysis, M.M.A.; Investigation, R.L.; Resources, P.M.T.; Data Curation, R.L. and R.J.; Writing-Original Draft Preparation, M.M.A.; Writing-Review \& Editing, M.M.A.; Supervision, M.M.A.; Project Administration and Funding Acquisition, P.M.T. and University Putra Malaysia

Funding: This research was funded by Ministry of Higher Education (KTP) and Higher Education Center of Excellence (HICoE), Malaysia, grant number: 6369109.

Acknowledgments: The authors would like to acknowledge the institute of tropical forestry and forest products (INTROP), and department of chemistry, faculty of science, Universiti Putra Malaysia (UPM) for supporting this project.

Conflicts of Interest: The authors declare no conflict of interest.

\section{References}

1. Nystrom, G.; Razaq, A.; Strømme, M.; Nyholm, L.; Mihranyan, A. Ultrafast All-Polymer Paper-Based Batteries. Nano Lett. 2009, 9, 3635-3639. [CrossRef] [PubMed]

2. Czaja, W.K.; Young, D.J.; Kawecki, M.; Brown, R.M., Jr. The Future Prospects of Microbial Cellulose in Biomedical Applications. Biomacromolecules 2007, 8, 1-12. [CrossRef] [PubMed]

3. Kim, J.; Yun, S.; Ounaies, Z. Discovery of Cellulose as a Smart Material. Macromolecules 2006, 39, $4202-4206$. [CrossRef]

4. Lee, A.D.H. Conductive Nanocrystalline Cellulose Polymer Composite Film as a Novel Mediator in Biosensor Applications. Master's Thesis, University of Toronto, Toronto, Canada, ON, December 2011.

5. Hoseini, S.H.; Entezami, A.A. Graft Copolymer of Polystyrene and Polypyrrole and Studies of Its Gas and Vapor Sensing Properties. Iran. Pol. J. 2005, 14, 101-110.

6. Ma, H.; Zhou, B.; Li, H.S.; Li, Y.Q.; Ou, S.Y. Green Composite Films Composed of Nanocrystalline Cellulose and a Cellulose Matrix Regenerated from Functionalized Ionic Liquid Solution. CarbohydPolymer 2011, 84, 383-389. [CrossRef]

7. Rawaida, L.R.; Abdi, M.M.; Tahir, P.M.; Moradbak, A.; Sulaiman, Y.; Lee, Y.H. Polyaniline-modified nanocellulose prepared from Semantan bamboo by chemical polymerization:preparation and characterization. RSC Adv. 2017, 7, 25191-25198.

8. Dubitsky, Y.A.; Zhubanov, B.A. Polypyrrole-Poly (Vinyl Chloride) and Polypyrrole-Cellulose Acetate Conducting Composite Films by Opposite-Diffusion Polymerization. Synt. Met. 1993, 53, 303-307. [CrossRef]

9. Hubbe, M.A. Method and Apparatus for Measuring an Electrical Property of Papermaking Furnish. U.S. Patent 5,936,151, 10 August 1999.

10. Nystrom, G.; Mihranyan, A.; Razaq, A.; Lindstrom, T.; Nyholm, L.; Strømme, M.A. Nanocellulose Polypyrrole Composite Based on Microfibrillated Cellulose from Wood. J. Phys. Chem. B 2010, 114, 4178-4182. [CrossRef] [PubMed]

11. Raba, J.; Mottola, H.A. Glucose Oxidase as an Analytical Reagent. Crit. Rev. Anal. Chem. 1995, $25,1-42$. [CrossRef] 
12. Zhang, D.; Zhang, L.; Wang, B.; Piao, G. Nanocomposite of polyaniline and cellulose nanocrystals prepared in lyotropic chiral nematic liquid crystals. J. Mater. 2013, 1-6. [CrossRef]

13. Takeo, S.; Hiroki, O.; Shuhei, S.; Kenichi, O.; Hiroyuki, N. Emerging N-Type Redox-Active Radical Polymer for a Totally Organic Polymer-Based Rechargeable Battery. Adv. Mater. 2009, 21, 1627-1630.

14. Dutta, D.; Sarma, T.K.; Chowdhury, D.; Chattopadhyay, A. A polyaniline-containing filter paper that acts as a sensor, acid, base, and endpoint indicator and also filters acids and bases. J. Colloid Interface Sci. 2005, 283, 153-159. [CrossRef] [PubMed]

15. Amalraj, J.; Suresha, K.M.; Jaehwan, K. The preparation, characterization and actuation behavior of polyaniline and cellulose blended electro-active paper. Smart Mater. Struct. 2010, 19, 045011.

16. Peng, B.L.; Dhar, N.; Liu, H.L.; Tam, K.C. Chemistry and applications of nanocrystalline cellulose and its derivatives: A nanotechnology perspective. Can. J. Chem. Eng. 2011, 89, 1191-1206. [CrossRef]

17. Liu, D.Y.; Sui, G.X.; Bhattacharyya, D. Synthesis and characterisation of nanocellulose-based polyaniline conducting films. Compos. Sci. Technol. 2014, 99, 31-36. [CrossRef]

18. Xia, L.; Wei, Z.; Wan, M. Conducting polymer nanostructures and their application in biosensors. J. Colloid Interface Sci. 2010, 341, 1-11. [CrossRef] [PubMed]

19. Viana, R.B.; Silva, B.F.; Pimentel, S. Infrared Spectroscopy of Anionic, Cationic, and Zwitterionic Surfactants. Adv. Phys. Chem. 2012, 903272. [CrossRef]

20. Dhyani, H.; Dhand, C.; Malhotra, B.D.; Sen, P. Polyaniline-CdS Quantum Dots Composite for Mediator Free Biosensing. Biosens. Bioelectron. 2011, 3, 112. [CrossRef]

21. Ghosh, S.; Vishalakshi, B.; Kalpagam, V. Polyaniline in the conducting state in neutral medium. Synth. Met. 1992, 46, 349. [CrossRef]

22. Upadhyay, J.; Kumar, A.; Gogoi, B.; Buragohain, A.K. Biocompatibility and antioxidant activity of polypyrrole nanotubes. Synth. Met. 2014, 189, 119-125. [CrossRef]

23. Nandini, S.; Nalini, S.; Reddy, M.B.M.; Suresh, G.S.; Melo, J.S.; Niranjana, P.; Shanmugam, S. Synthesis of one-dimensional gold nanostructures and the electrochemical application of the nanohybrid containing functionalized graphene oxide for cholesterol biosensing. Bioelectrochemistry 2016, 110, 79-90. [CrossRef] [PubMed]

24. Alam, M.; Ansari, A.A.; Rafi, M.; Alandis, N.M. Optical and electrical conducting properties of Polyaniline/Tin oxide nanocomposite. ARAB J. Chem. 2013, 6, 341-345. [CrossRef]

25. Khairkar, S.R.; Raut, A.R. Synthesis of Chitosan-graft-Polyaniline-Based Composites. AJMSE 2014, 4, 62-67. [CrossRef]

26. Martone, P.T.; Estevez, J.M.; Lu, F.; Ruel, K.; Denny, M.W.; Somerville, C.; Ralph, J. Discovery of lignin in seaweed reveals convergent evolution of cell wall architecture. Curr. Biol. 2009, 19, 169-175. [CrossRef] [PubMed]

27. Barik, A.; Solanki, P.R.; Kaushik, A. Polyanilinecarboxymethyl cellulose nanocomposite for cholesterol detection. J. Nanosci. Nanotechnol. 2010, 10, 6479-6488. [CrossRef] [PubMed]

28. Luong, N.D.; Korhonen, J.T.; Soininen, A.J.; Ruokolainen, J.; Johansson, L.S.; Seppälä, J. Processable polyaniline suspensions through in situ polymerization onto nanocellulose. Eur. Polym. J. 2013, 49, 335-344. [CrossRef]

29. Zhou, Y.; Song, Q.; Liang, B.; Qin, Z. Preparation and Capacitive Properties of Polyaniline/Nanocellulose Composites with Core-Shell Structure. In Proceedings of the International Conference on Advances in Energy, Environment and Chemical Engineering, Changsha, China, 26-27 September 2015.

30. Ede, S.R.; Nithiyanantham, U.; Kundu, S. DNA-encapsulated chain and wire-like $\beta-\mathrm{MnO}_{2}$ organosol for oxidative polymerization of pyrrole to polypyrrole. Phys. Chem. Chem. Phys. 2015, 17, 5474-5484. [CrossRef] [PubMed]

31. Murugan, A.V.; Muraliganth, T.; Manthiram, A. Rapid, facile microwave-solvothermal synthesis of graphene nanosheets and their polyaniline nanocomposites for energy strorage. Chem. Mater. 2009, 21, 5004-5006. [CrossRef]

32. Zhang, X.; Zhang, J.; Song, W.; Liu, Z. Controllable Synthesis of Conducting Polypyrrole Nanostructures. J. Phys. Chem. 2006, 110, 1158-1165. [CrossRef] [PubMed]

33. Ayad, M.; El-Hefnawy, G.; Zaghlol, S. Facile synthesis of polyaniline nanoparticles; its adsorption behavior. Chem. Eng. J. 2013, 217, 460-465. [CrossRef] 
34. Kondawar, S.B.; Deshpande, M.D.; Agrawal, S.P. Transport Properties of Conductive Polyaniline Nanocomposites Based on Carbon Nanotubes. J. Compos. Mater. 2012, 2, 32-36. [CrossRef]

35. Terinte, N.; Ibbett, R.; Schuster, K.C. Overview on native cellulose and microcrystalline cellulose I structure studied by X-ray diffraction (WAXD): Comparison between measurement techniques. Lenzinger Berichte 2011, 89, 118-131.

36. Joelma, M.; Cardoso, R.; Fogliato, M.; Lima, S.; Lenz, D.M. Polyaniline Synthesized with Functionalized Sulfonic Acids for Blends Manufacture. Mater. Res. 2007, 10, 425-429.

37. Zhang, X.; Zhu, J.; Haldolaarachchige, N.; Ryu, J.; Young, D.P.; Wei, S.; Guo, Z. Synthetic process engineered polyaniline nanostructures with tunable morphology and physical properties. Polymer 2012, 53, 2109-2120. [CrossRef]

38. Casado, U.M.; Aranguren, M.I.; Marcovich, N.E. Preparation and characterization of conductive nanostructured particles based on polyaniline and cellulose nanofibers. Ultrason. Sonochem. 2014, 21, 1641-1648. [CrossRef] [PubMed]

39. Mo, Z.; Zhao, Z.; Chen, H.; Niu, G.; Shi, H. Heterogeneous preparation of cellulose-polyaniline conductive composites with cellulose activated by acids and its electrical properties. Carbohydr. Polym. 2009, 75, 660-664. [CrossRef]

40. Goworek, J.; Kierys, A.; Gac, W.; Borówka, A.; Kusak, R. Thermal degradation of CTAB in as-synthesized MCM-41. J. Therm. Anal. Calorim. 2009, 96, 375-382. [CrossRef]

Sample Availability: Samples of the compounds are available from the authors.

(C) 2018 by the authors. Licensee MDPI, Basel, Switzerland. This article is an open access article distributed under the terms and conditions of the Creative Commons Attribution (CC BY) license (http://creativecommons.org/licenses/by/4.0/). 